\title{
Caracterização da fauna helmintológica de tamanduá-bandeira (Myrmecophaga tridactyla) e tamanduá-mirim (Tamandua tetradactyla) atropelados nas rodovias BR-050 e BR-455 (Minas Gerais, Brasil)
}

\author{
[Helmintological characterization of giant anteater (Myrmecophaga tridactyla) and lesser \\ anteater (Tamandua tetradactyla) roadkilled on BR-050 and BR-455 \\ highways (Minas Gerais, Brazil)]
}

\author{
W.J. Oliveira ${ }^{1}$, A.L.Q. Santos ${ }^{2}$, W.V. Souza $a^{3}$, A.E.I. Custódio ${ }^{2}$, \\ E.G. Lux Hoppe ${ }^{4}$, J.H. Tebaldi ${ }^{4}$, F. Rosalinski Moraes ${ }^{2}$
}

\author{
${ }^{1}$ Aluno de pós-graduação - Universidade Estadual Paulista Júlio de Mesquita Filho - Jaboticabal, SP \\ ${ }^{2}$ Universidade Federal de Uberlândia - Uberlândia, MG \\ ${ }^{3}$ Aluno de pós-graduação - Universidade Federal de Uberlândia - Uberlândia, MG \\ ${ }^{4}$ Universidade Estadual Paulista Júlio de Mesquita Filho - Jaboticabal, SP
}

\begin{abstract}
RESUMO
O tamanduá-bandeira (Myrmecophaga tridactyla) e o tamanduá-mirim (Tamandua tetradactyla) são mamíferos xenartros amplamente distribuídos no território nacional. Apesar disso, suas populações estão em constante ameaça, principalmente o primeiro, categorizado como vulnerável de acordo com classificação da União Internacional para Conservação da Natureza. Diante disso e da escassez de estudos com endoparasitas nesses animais, objetivou-se descrever a helmintofauna dessas duas espécies de tamanduá, utilizando espécimes atropelados nas rodovias BR-050 e BR-455, região do Triângulo Mineiro, Minas Gerais, Brasil. Os animais foram submetidos à necropsia, tendo seus órgãos e conteúdo intestinal e estomacal examinados para recuperação de helmintos. Os parasitos coletados foram identificados com base em suas características morfológicas e morfométricas. As espécies/os gêneros caracterizados para $M$. tridactyla e T. tetradactyla, suas respectivas prevalências, bem como órgãos de origem, foram: Gaphidiops dissimilis (33,33\% e 0\%) no estômago; Gigantorhynchus echinodiscus $(25 \%$ e 66,66\%) no intestino delgado; Physaloptera magnipapilla (50\% e 66,6\%) no estômago; e Mathevotaenia spp. (25\% e 66,66\%) no intestino delgado. Physaloptera magnipapilla foi a espécie com os maiores índices de infecção, e novo registro de localização foi registrado para o nematódeo Graphidiops dissimilis. Detectou-se uma provável sobreposição da área de vida dos tamanduás estudados, bem como uma helmintofauna característica da região.
\end{abstract}

Palavras-chave: tamanduá, necropsia, helmintos

\begin{abstract}
Myrmecophaga tridactyla, giant anteater and Tamandua tetradactyla, collared anteater are nationally widespread mammals. Despite that, their populations are in danger, especially the first one, categorized as vulnerable according to the International Union for Conservation of Nature. This study reports the occurrence of helminths in these two anteater species using road killed specimens from BR-050 and BR455 highways, Triângulo Mineiro region, Minas Gerais state, Brazil. The species were necropsied, and their gastrointestinal organs and contents were opened and examined for parasite recovery. The identification was based on morphological and morphometric features. The species/ genera found for $\mathrm{M}$. tridactyla and T. tetradactyla, their prevalence, as well the organs, were: Gaphidiops dissimilis $(33.33 \%$ and $0 \%)$ in stomach; Gigantorhynchus echinodiscus (25\% and 66.66\%) in small intestine; Physaloptera magnipapilla $(50 \%$ and $66.6 \%)$ in stomach and Mathevotaenia spp. $(25 \%$ and $66.66 \%)$ in small intestine. Physaloptera magnipapilla was the most common species and a new location was reported for Graphidiops
\end{abstract}

Recebido em 10 de dezembro de 2019

Aceito em 28 de abril de 2020

E-mail:wilsonjr2009@hotmail.com 
dissimilis. There is a probable overlap in the home range of these animals and a local helminth diversity was described on the study site.

Keywords: anteater, necropsy, helminths

\section{INTRODUÇÃO}

O tamanduá-bandeira (Myrmecophaga tridactyla) e o tamanduá-mirim (Tamandua tetradactyla) são mamíferos que podem ser encontrados em todo o território nacional, além de áreas como o norte do Uruguai, na Argentina, na Venezuela, na Colômbia e nas Guianas (Medri et al., 2011; Miranda, 2014). A sobrevivência desses animais é constantemente ameaçada por ataques de cães, queimadas, caça predatória e fragmentação de seu hábitat (Superina et al., 2010). É importante ressaltar que o tamanduá-bandeira figura na lista de espécies brasileiras ameaçadas de extinção, classificado como vulnerável na lista da União Internacional para Conservação da Natureza (IUCN), demandando esforços crescentes para a conservação da espécie no país (Miranda et al., 2015).

Outra causa de mortalidade desses animais, principalmente de tamanduás-mirins, é o atropelamento em rodovias, que pode superar inclusive os impactos causados pela caça ou até mesmo mortes por causa natural (Valadão et al., 2018). O comportamento letárgico e a característica dessas espécies de percorrer longas distâncias à procura de alimento aumentam seus riscos de colisão com veículos (Pinto et al., 2018). As rodovias podem ainda constituir uma barreira física e prejudicar a movimentação, o acesso a recursos e o fluxo genético desses indivíduos, contribuindo ainda mais para a diminuição de seus exemplares (Chen e Koprowski, 2016).

$\mathrm{O}$ estudo da diversidade de helmintos em populações de animais silvestres é uma importante contribuição para a conservação dessas espécies, pois revela a condição de saúde dos ecossistemas por meio de mudanças na relação de simbiose parasito- hospedeiro, podendo, assim, auxiliar conservacionistas no acompanhamento de mudanças ambientais que ameacem a sobrevivência desses animais (Lymbery, 2005). A identificação desses parasitos pode ainda fornecer subsídios para a realização de estudos posteriores que avaliem suas características patogênicas e possível impacto nas populações de animais silvestres estudadas.
Diante desse contexto, da escassez de estudos sobre a ocorrência de endoparasitos em $M$. tridactyla e $T$. tetradactyla e do pouco aproveitamento de carcaças de animais atropelados para estudos helmintológicos (Griese, 2007; Rojano et al., 2015), objetiva-se caracterizar a fauna helmintológica de tamanduábandeira (Myrmecophaga tridactyla) e tamanduámirim (Tamandua tetradactyla) atropelados nas rodovias BR-050 e BR-455, região do Triângulo Mineiro, Minas Gerais.

\section{MATERIAL E MÉTODOS}

Sete tamanduás adultos (quatro tamanduás-mirins e três tamanduás-bandeira) foram recuperados em trechos das rodovias BR-050 e BR-455, ambas situadas na região do Triângulo Mineiro, MG. Os animais utilizados fazem parte de um estudo anterior que monitorou o atropelamento de espécies selvagens nesses locais. A BR-050 (Fig. 1) é uma rodovia federal brasileira, que se inicia na cidade de Brasília (DF) e termina em Santos (SP), passando pelo Distrito Federal e pelos estados de Goiás, Minas Gerais e São Paulo. Com uma extensão de $1.025,3 \mathrm{~km}$, é considerada uma das rodovias mais movimentadas do país, pois liga a capital federal a São Paulo. Parte da rodovia encontra-se sob jurisdição dos governos estaduais e opera por concessionárias privadas. Nestes trechos, a BR-050 apresenta pista dupla e pedágios.

Por tal motivo, nestes trechos, a rodovia perde a nomenclatura original e passa a ser integrante da malha viária do estado onde está localizada. Serve, entre outras, as seguintes cidades: Cristalina (GO), Catalão (GO), Araguari (MG), Uberlândia (MG), Uberaba (MG) e Cumari (GO) (Brasil, 2019a). A BR-455 (Fig. 2) é uma rodovia federal de ligação brasileira. Inicia-se em Uberlândia, Minas Gerais, e termina na rodovia BR-364, município de Planura, próximo à divisa com São Paulo. Passa ainda pelos municípios de Prata, Campo Florido e Pirajuba, todos em Minas Gerais. Possui extensão de 137,3km (Brasil, 2019b). 
A região do Triângulo Mineiro apresenta população em torno de 1.500 .000 habitantes e é constituída por cinco principais cidades: Uberlândia, Uberaba, Patos de Minas, Araguari e Ituiutaba. O clima é do tipo Aw, segundo classificação de Köppen (1948), apresentando sazonalidade com chuvas no verão e seca no inverno, com temperaturas médias entre $17^{\circ} \mathrm{C}$ e $23^{\circ} \mathrm{C}$ e amplitude térmica anual entre $7^{\circ} \mathrm{C}$ e $9^{\circ} \mathrm{C}$ (Rosa et al., 1991).

A área de estudo está inserida no bioma Cerrado. Atualmente, as formações naturais da região estão restritas a fragmentos, devido à pressão exercida pelas atividades agropecuárias, notadamente monocultura canavieira e pastagens (Carvalho et al., 2015). As carcaças foram acondicionadas em freezers a $-20^{\circ} \mathrm{C}$ até o devido processamento. Os animais foram descongelados em temperatura ambiente e submetidos à necropsia no Laboratório de Pesquisa em Animais Silvestres (Lapas) da Universidade Federal de Uberlândia, entre outubro de 2018 e fevereiro de 2019.

As porções gastrointestinais (estômago, duodeno, jejuno, íleo, ceco, cólon e reto) foram retiradas, individualizadas mediante amarração com barbantes e abertas com o auxílio de uma tesoura fina-fina. $\mathrm{O}$ conteúdo gastrointestinal foi lavado em água corrente, tamisado em peneira com malha metálica de abertura de 100 micrômetros, acondicionado em frascos plásticos e encaminhado para o Laboratório de Doenças Parasitárias, onde foi observado com o auxílio de microscópio estereoscópio para coleta de helmintos. Todos os helmintos recuperados foram clarificados com ácido acético $80 \%$ e, caso necessário, com creosoto de faia e identificados de acordo com Anderson et al. (2009); Vicente et al.
(1997) e Ortlepp (1922) para nematódeos; Yamaguti (1963) para acantocéfalos; e Khalil et al. (1994) para cestódeos. Características morfológicas, morfométricas e imagens foram obtidas com um microscópio Olympus BX-51 acoplado a uma câmera Q Color 3 (Olympus, Tóquio, Japão) e processadas por meio do software ImagePro Plus v. 4.0.

Os indicadores de prevalência, intensidade parasitária média (IPM), abundância e variação de intensidade foram calculados conforme Bush et al. (1997). Todos os procedimentos adotados foram avaliados pelo Comitê de Ética na Utilização de Animais (Ceua/UFU, protocolo $n^{\circ}$ A015/19) e pelo Instituto Chico Mendes de Conservação de Biodiversidade (Sisbio, protocolo 70460-1)

\section{RESULTADOS}

Um total de 93 helmintos foi coletado. Um dos tamanduás-mirins analisados não apresentou parasitismo por qualquer espécie de helminto. As espécies/os gêneros de parasitos recuperados em Tamandua tetradactyla foram: Physaloptera magnipapilla, Mathevotaenia spp. e Gigantorhynchus echinodiscus. Já em Myrmecophaga tridactyla, as espécies Graphidiops dissimilis, Physaloptera magnipapilla, Mathevotaenia spp. e Gigantorhynchus echinodiscus foram observadas. As prevalências variaram entre $25 \%$ e $50 \%$ para Tamandua tetradactyla e de $33,33 \%$ a $66,66 \%$ para Myrmecophaga tridactyla, sendo o nematódeo Physaloptera magnipapilla o helminto mais frequente e com maior intensidade parasitária para ambas as espécies de tamanduá analisadas (Tab. 1 e 2).

Tabela 1. Indicadores de infecção observados em Tamandua tetradactyla atropelados nas rodovias BR-050 e BR-455, na região do Triângulo Mineiro, Minas Gerais, IPM - intensidade parasitária média

\begin{tabular}{lcccc}
\multicolumn{1}{c}{ Helmintos } & Prevalência & IPM & Abundância & $\begin{array}{c}\text { Variação de } \\
\text { intensidade }\end{array}$ \\
\hline $\begin{array}{l}\text { NEMATODA } \\
\text { Physalopteridae } \\
\begin{array}{l}\text { Physaloptera magnipapilla } \\
\text { CESTODA }\end{array}\end{array}$ & $50 \%(2 / 4)$ & 7 & 3,5 & $3-11$ \\
$\begin{array}{l}\text { Anoplocephalidae } \\
\text { Mathevotaenia spp. }\end{array}$ & $25 \%(1 / 4)$ & 4 & 0,25 & - \\
$\begin{array}{l}\text { ACANTOCEPHALA } \\
\text { Gigantorhyncidae } \\
\text { Gigantorhynchus echinodiscus }\end{array}$ & $25 \%(1 / 4)$ & 1 & 1 & - \\
\hline
\end{tabular}


Oliveira et al.

Tabela 2. Indicadores de infecção observados em Myrmecophaga tridactyla atropelados nas rodovias BR050 e BR-455, na região do Triângulo Mineiro, Minas Gerais

\begin{tabular}{lcccc}
\multicolumn{1}{c}{ Helmintos } & Prevalência & IPM & Abundância & $\begin{array}{c}\text { Variação de } \\
\text { intensidade }\end{array}$ \\
\hline $\begin{array}{l}\text { NEMATODA } \\
\text { Molineidae } \\
\text { Anoplostrongylinae }\end{array}$ & & & & \\
$\begin{array}{l}\text { Graphidiops dissimilis } \\
\text { Physalopteridae }\end{array}$ & $33,33 \%(1 / 3)$ & 6 & 2 & - \\
$\begin{array}{l}\text { Physaloptera magnipapilla } \\
\text { CESTODA } \\
\quad \text { Anoplocephalidae }\end{array}$ & $66,66 \%(2 / 3)$ & 29 & 19,33 & $25-33$ \\
$\begin{array}{c}\text { Mathevotaenia } \text { spp. } \\
\text { ACANTOCEPHALA } \\
\text { Gigantorhynchus echinodiscus }\end{array}$ & $66,66 \%(2 / 3)$ & 4,5 & 3 & $3-6$ \\
\hline IPM ing & $66,66 \%(2 / 3)$ & 9,5 & 6,33 & $1-18$ \\
\hline
\end{tabular}

IPM - intensidade parasitária média.

Graphidiops dissimilis - Vicente et al. (1997) Fig. 1 (A,B,C,D,E,F)

Descrição geral: Tais nematódeos são pequenos, finos, com corpo apresentando linhas longitudinais salientes. Sua extremidade anterior possui dilatação cuticular cefálica pouco proeminente. A boca é composta por três lábios com presença de papilas diminutas. Esôfago claviforme. $\mathrm{O}$ anel nervoso situa-se ao nível do início da porção glandular do esôfago, próximo ao poro excretor. As fêmeas são didelfas, anfidelfas e com tendência à prodelfia; seu ovejetor é forte $\mathrm{e}$ com ramos divergentes e ovos de casca fina, lisa e morulados estão presentes no útero. Os machos possuem bolsa copulatória trilobada, simétrica e do tipo 2-1-2. Presença de papilas pré-bursais. Fórmula bursal: raios laterais 2 e 3 nascem de um tronco comum e são contíguos, da mesma maneira dos raios laterais 5 e 6 , atingindo a margem da bolsa. O raio lateral 4 nasce isolado, sendo o mais curto dos raios do lobo lateral, não atingindo a margem da bolsa. As bordas dos lobos laterais e dos raios dorsais são espessas. Raios dorsais externos nascem da base do raio dorsal e se dirigem para lados opostos. Raio dorsal termina em duas pontas bífidas próximas à margem bursal. Os espículos são relativamente longos e quitinizados, terminando em duas pontas em sua porção distal. Presença de gubernáculo. A cauda termina em ponta fina.

Dados morfométricos - Machos ( $\mathrm{n}=3$ ): Possuem comprimento total de $8,95 \pm 0,98(9,93-7,60)$; o esôfago mede $0,76 \pm 0,043(0,80-0,70)$; sua largura (junção esôfago-intestinal) é de $0,08 \pm$ $0,006(0,09-0,07)$; o anel nervoso possui $0,40 \pm$ $0,054(0,46-0,33)$; a medida do poro excretor é de $0,38 \pm 0,023(0,40-0,35)$; os espículos medem
$0,61 \pm 0,087(0,68-0,49)$. Fêmeas $(n=3)$ : Seu comprimento total é de $12,88 \pm 0,466(13,42-$ $12,28)$; o esôfago mede $0,82 \pm 0,028(0,86-0,80)$; possuem largura (junção esôfago-intestinal) de $0,12 \pm 0,008(0,13-0,11)$; o anel nervoso mede $0,37 \pm 0,016(0,39-0,35)$; o poro excretor mede $0,46 \pm 0,012(0,48-0,45)$; a distância da vulva até a extremidade posterior é de 2,15 $\pm 0,071(2,11$ $-2,09)$; o ovejetor tem $0,43 \pm 0,032(2,11-2,09)$; os ovos são de $0,051 \pm 0,003(0,056-0,048) \mathrm{x}$ $0,023 \pm 0,002(0,027-0,021)$.

\section{Physaloptera magnipapilla - Ortlepp (1922)}

Fig. 2 (A,B,C,D,E)

Descrição geral: $O$ helminto dispõe de cutícula espessa, com leves estriações transversais, cujo comprimento por vezes ultrapassa os lábios e envolve a extremidade anterior à semelhança de um colarete. Os lábios são constituídos por um dente externo com ponta quadrangular e um dente interno tripartite. A papila cervical é arredondada e próxima à abertura do poro excretor. Esôfago reto. As fêmeas apresentam abertura vulvar próxima à região média do corpo e ovos pequenos, de casca grossa e embrionados estão presentes no útero. Os machos possuem asa caudal com quatro pares de papilas pedunculares equidistantes, três papilas pré-anais, dois pares de papilas situadas próximo ao ânus, um outro par disposto de forma oblíqua em uma localização posterior, e quatro papilas dispostas em pares se aproximando do final da cauda. Os espículos são pontiagudos, curvados e de tamanhos semelhantes. A cauda termina em projeção pontiaguda.

Dados morfométricos - Machos $(\mathrm{n}=5)$ : Detém comprimento total de 26,61 $\pm 8,93(34,02-$ 


\section{Caracterização da fauna...}

12,07); sua papila cervical mede $1,71 \pm 1,31(3,41$ $-0,43)$; o poro excretor possui $1,91 \pm 1,38(3,65$ $-0,52)$; o anel nervoso mede 1,61 $\pm 1,08$ (3,02 $0,23)$; o esôfago apresenta $6,51 \pm 2,85(8,98-$ 2,01); possuem largura (junção esôfagointestinal) de $0,85 \pm 0,27(1,04-0,38)$; o espículo maior é de $1,01 \pm 0,65(2,17-0,59)$; e o espículo menor tem por comprimento $0,89 \pm 0,55(1,84-$ $0,40)$. Fêmeas $(\mathrm{n}=5)$ : Mantém comprimento total de $37,07 \pm 1,66(38,44-35,22)$; sua papila
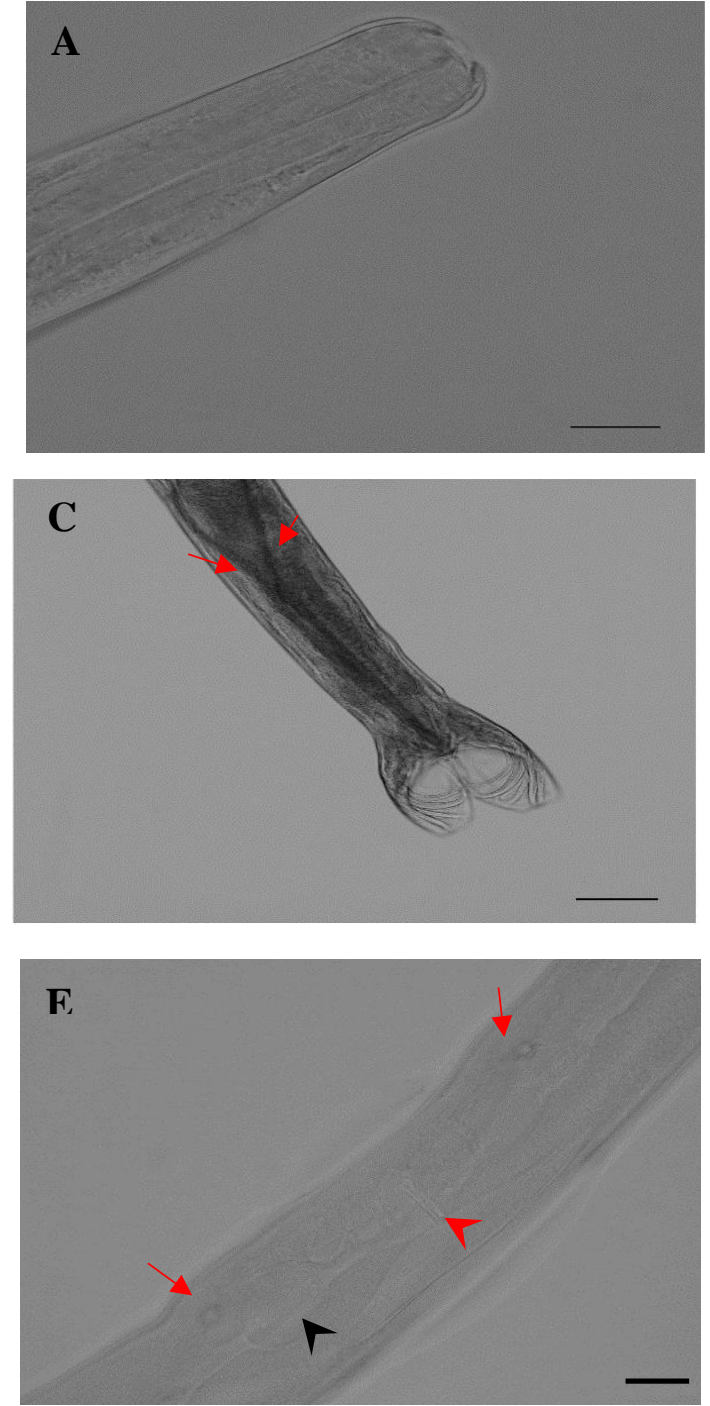

Figura 1. Graphidiops dissimilis recuperado do estômago de Myrmecophaga tridactyla. A: extremidade anterior. Aumento: 400x. Escala: $20 \mu \mathrm{m}$. B: Abertura do poro excretor (seta vermelha). Aumento: 400x. Escala: $20 \mu \mathrm{m}$. C: Extremidade posterior do macho, evidenciando os longos espículos (setas vermelhas).

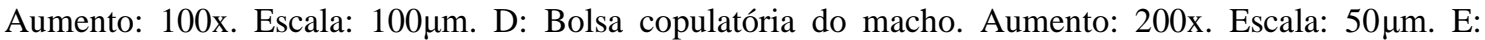
Estruturas reprodutivas presentes na fêmea, evidenciando a localização da vulva (ponta de seta vermelha),

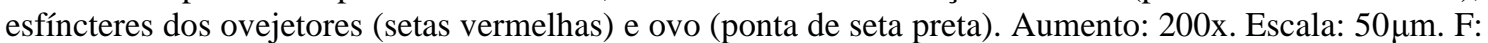
Extremidade posterior da fêmea. Aumento: 100x. Escala: $100 \mu \mathrm{m}$. 


\section{Oliveira et al.}
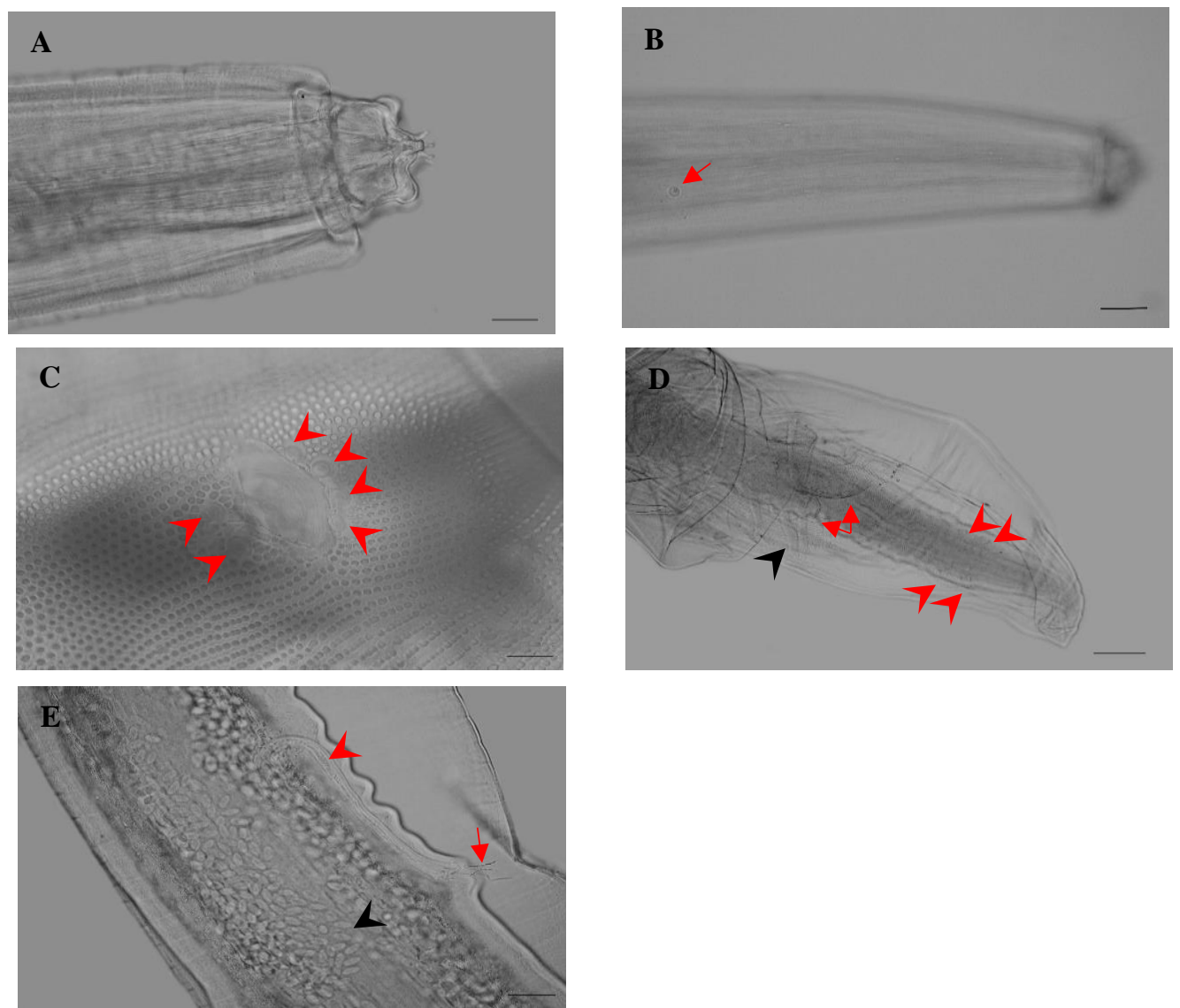

Figura 2. Physaloptera magnipapilla recuperada do estômago de Myrmecophaga tridactyla e Tamandua

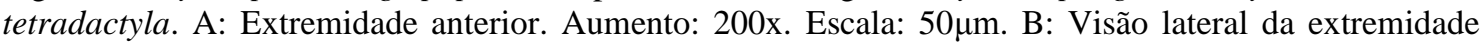
anterior, com destaque para a papila cervical (seta vermelha). Aumento: 100x. Escala: 100 $\mu$ m. C: Ânus e papilas cloacais do macho (pontas de seta). Aumento: 200x. Escala: 50 $\mu \mathrm{m}$. D: Asa caudal do macho, evidenciando algumas papilas pedunculadas (ponta de seta preta) e papilas pós-cloacais (pontas de seta vermelha), além dos espículos (setas vermelhas). Aumento: 40x. Escala: 2000 $\mu \mathrm{m}$. E: Estruturas reprodutivas da fêmea, destacando a abertura da vulva (seta vermelha), ovejetor (ponta de seta vermelha) e ovos (ponta de seta preta). Aumento: 100x. Escala: $100 \mu \mathrm{m}$.

Gigantorhynchus echinodiscus - Lent e Freitas (1938) - Figura 3 (A, B, C, D, E,F,G)

Descrição geral: $\mathrm{O}$ acantocéfalo é longo, de tamanho variável e pseudossegmentado. Tal helminto possui probóscide cilíndrica com coroa única ou dupla no ápice (composta por seis ganchos maiores) e diversas fileiras de 18 ganchos menores distribuídos ao longo do restante da tromba. Seus lemniscos são filiformes e alongados, atingindo a metade do tronco. O aparelho reprodutivo do macho é constituído por um par de testículos elipsoides dispostos em tandem, oito glândulas do cemento em fileira única, seguida por longo ducto ejaculador e bolsa copulatória por vezes retraída. A extremidade posterior da fêmea termina em uma campânula onde estão localizados o útero, a vagina e o poro genital. Os ovos, localizados no útero, são elípticos e compostos por três membranas.

Machos ( $\mathrm{n}=2)$ : Possuem comprimento total de 29 $\pm 70,71(34-24)$; os ganchos apicais medem 0,18 $\pm 0,02(0,20-0,17) \times 0,04 \pm 0,007(0,04-0,05)$; e demais ganchos $0,04 \pm 0,01(0,06-0,03) \times 0,03$ $\pm 0,01(0,05-0,02)$; seu prossoma tem por medida $1,79 \pm 0,14(1,89-1,69)$; os lemniscos detém $14,37 \pm 0,68(13,89-14,85)$; o testículo anterior mede $2,48 \pm 0,31(2,7-2,26) \times 0,6 \pm 0,03$ $(0,62-0,58)$; já o testículo posterior possui 2,68 $\pm 0,45(3-2,36) \times 0,56 \pm 0,01(0,57-0,55)$; as glândulas do cemento tem $0,67 \pm 0,08(0,74-$ $0,53) \times 0,56 \pm 0,09(0,66-0,43)$ e seu conjunto 


\section{Caracterização da fauna...}

$4,39 \pm 0,04(4,36-4,42)$. Fêmeas $(n=2)$ : Seu comprimento total é de $302,31 \pm 353,91(552,56$ - 52,05); possuem ganchos apicais com $0,18 \pm$ $0,02(0,20-0,17) \times 0,04 \pm 0,007(0,04-0,05)$; e demais ganchos com $0,03 \times 0,03 \pm 0,01(0,05-$ $0,03)$; seu prossoma mede $1,59 \pm 0,06(1,63-$ 1,54); já os lemniscos tem por comprimento 14,21
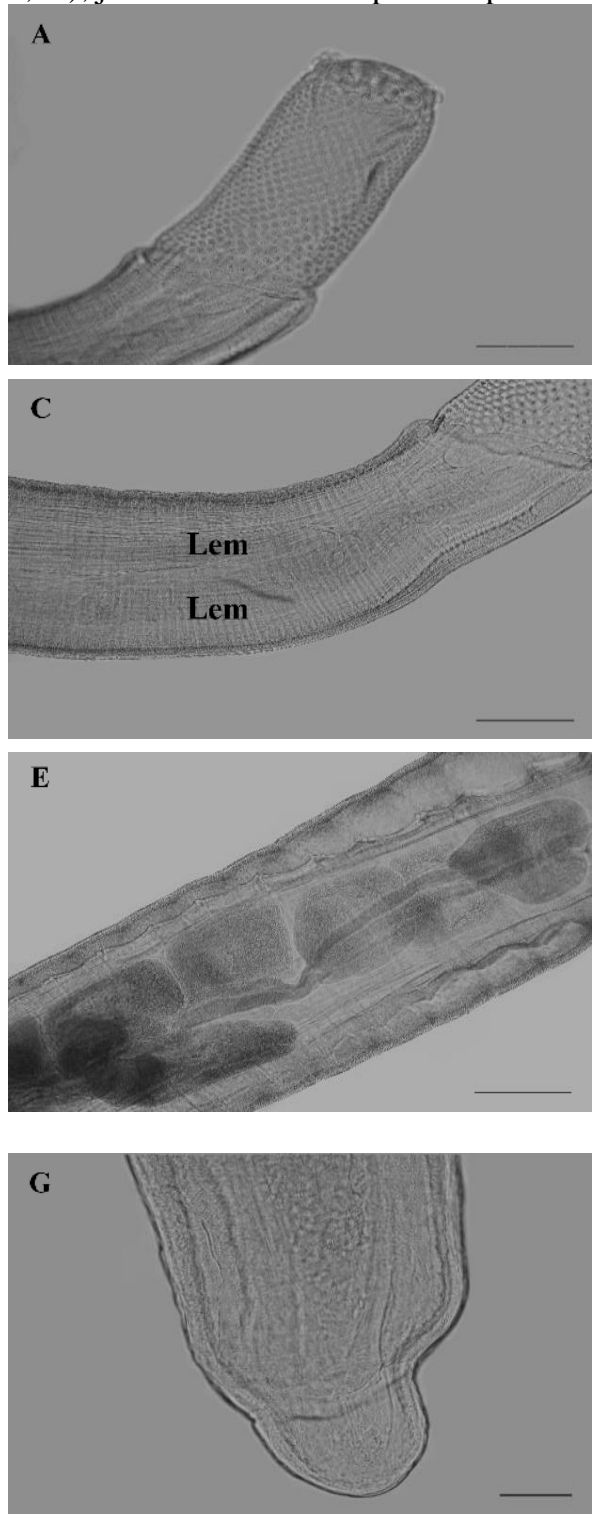

Figura 3. Gigantorhynchus echinodiscus recuperado do intestino delgado de Myrmecophaga tridactyla e Tamandua tetradactyla. A: Extremidade anterior. Aumento 40x. Escala: 2000 $\mu \mathrm{m}$. B: Secção transversal da probóscide armada com 18 ganchos. Aumento: 100x. Escala: 50 $\mu \mathrm{m}$. C: Lemniscos (Lem) longos e largos.

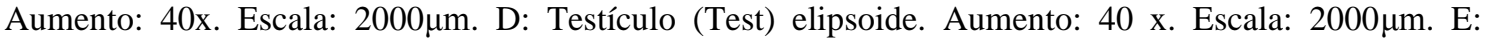

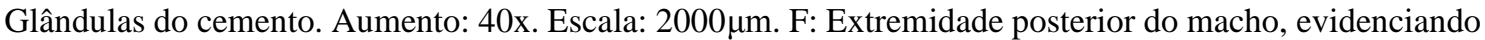
bolsa copulatória (Bc) retraída. Aumento: 40x. Escala: $2000 \mu \mathrm{m}$. G: Extremidade posterior da fêmea. Aumento: 40x. Escala: $2000 \mu \mathrm{m}$. 


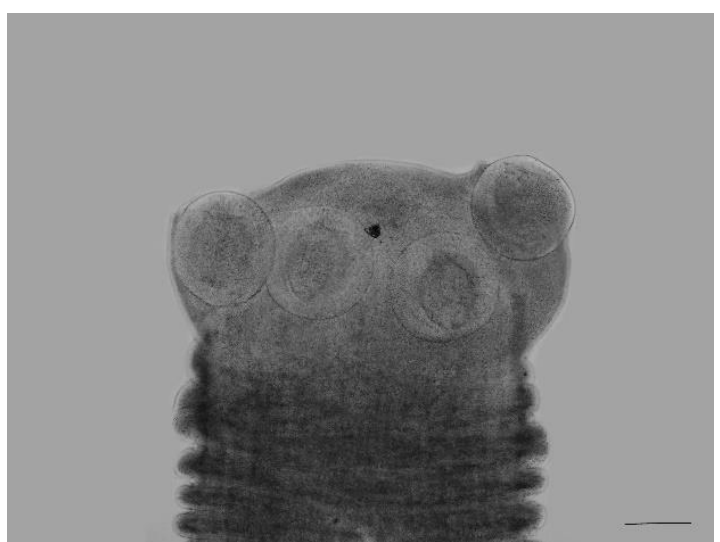

Figura 4. Mathevotaenia spp. recuperada do intestino delgado de Myrmecophaga tridactyla e Tamandua tetradactyla. Escólex com quatro ventosas sem rostelo e proglotes craspedotas. Aumento: 40x. Escala: 2000um.

\section{DISCUSSÃO}

O gênero Gaphidiops spp. compreende as espécies G. costalimai (Lent e Freitas, 1938); G. inaequalis (Lent e Freitas, 1938); G. cyclopi (Diaw, 1976); G. major (Travassos, 1949); G. ruschii (Travassos, 1949); G. assimilis (Freitas e Mendonça, 1959); G. dissimilis (Freitas e Mendonça, 1959) e G. browni (Cameron e Myers, 1961) descritas parasitando o estômago e o intestino de tamanduás (Myrmecophaga tridactyla, Tamandua tetradactyla e Cyclopes didactylus) no Brasil, nos Estados Unidos e na França. A espécie encontrada no presente estudo, Graphidiops dissimilis, diferencia-se das demais pela presença de pontas bífidas nos espículos dos machos, espessamentos cuticulares transversais e asas perivulvares nas fêmeas, além de diferentes características morfométricas (Freitas e Mendonça, 1959). O tricostrongilídeo foi anteriormente relatado no estado do Mato Grosso, constituindo, assim, de acordo com os dados da presente pesquisa, novo registro de localização.

Physaloptera spp. é um nematódeo presente em diversas espécies de aves, répteis, anfíbios e mamíferos (Ortlepp, 1922). Já foram reportadas, no Brasil, cerca de 13 espécies do gênero, sendo que $P$. magnipapilla, $P$. papillotruncata, $P$. semilanceolata e $P$. praeputialis estavam presentes no estômago de Myrmecophaga tridactyla e Tamandua tetradactyla (Vicente et al., 1997; Griese, 2007; Ramos et al., 2016). Physaloptera magnipapilla foi um achado frequente, com as maiores prevalências $\mathrm{e}$ intensidades parasitárias entre os helmintos recuperados, sendo identificada principalmente pela morfologia e distribuição das papilas pré e pós-anais presentes na asa caudal do macho. Tal parasita é hematófago e pode causar lesões gástricas hiperplásicas e anemia, perda de peso, vômito e diarreia nos animais acometidos (Clark, 1990; Lértora et al., 2016), no entanto não foram observadas lesões gástricas aparentes nos animais submetidos à necropsia, sendo necessários outros estudos para avaliação da extensão e gravidade das lesões causadas por esse helminto.

O gênero Gigantorhynchus foi originalmente descrito por Hamann (1892) e inclui seis espécies parasitas de tamanduás, babuínos e marsupiais (Amin, 2013). No Brasil, há registros de G. lutzi em Caluromys philander (Machado Filho, 1941) e G. echinodiscus em Myrmecophaga tridactyla, Tamandua tetradactyla e Cyclopes didactylus (Yamaguti, 1963). Gigantorhynchus echinodiscus, o acantocéfalo recuperado no estudo, teve sua identificação baseada na quantidade e na disposição dos ganchos da probóscide, no formato e no número dos testículos e nas glândulas do cemento do macho, além dos lemniscos grandes e filiformes. As características morfométricas dos espécimes recuperados assemelham-se às descritas por Gomes et al. (2019) e Yamaguti (1963).

Os cestódeos encontrados no estudo, pertencentes ao gênero Mathevotaenia spp., são comumente observados em mamíferos (marsupiais, xenartros, carnívoros, quirópteros, roedores e primatas), com relatos pontuais em répteis e aves (Lunaschi et al., 2012). Na América do Sul, identificaram-se 
15 espécies no intestino delgado de diversos animais, com M. tetragonocephala descrita por Schmidt e Martin (1978) em Myrmecophaga tridactyla e Tamandua tetradactyla. As descrições morfológicas de cestódeos recuperados por Griese (2007) nessas duas espécies de tamanduá aproximam-se de $M$. surinamensis, apontando a espécie como constituinte de sua helmintofauna. A presença de quatro ventosas no escólex com ausência de rostelo e proglotes craspedotas foi suficiente para a classificação do cestódeo em Mathevotaenia spp., não sendo possível sua caracterização em menor táxon pela ausência de proglotes maduras e, por conseguinte, a descrição das estruturas reprodutivas. Inferências podem ser feitas quanto a M. tetragonocephala, já que Frank et al. (2012) encontraram o platelminto na mesma região geográfica do presente estudo.

O ciclo de vida da maioria dos helmintos recuperados tem artrópodes como hospedeiros intermediários, tais como cupins para Gigantorhynchus echinodiscus (Amato et al., 2014); besouros, grilos e baratas para Physaloptera spp. (Lértora et al., 2016) e baratas e borboletas para Mathevotaenia spp. (Spasskii, 1951). Dessa forma, os hábitos alimentares dos tamanduás, predominantemente insetívoros, constituem importante forma de transmissão de endoparasitoses, principalmente quando envolvem ciclos heteroxênicos (Rojano et al., 2015). O contato com o solo, a ingestão acidental de material vegetal e de água contaminada podem contribuir para a existência de parasitoses monoxênicas, como os tricostrongilídeos do gênero Graphidiops spp. (Ezquiaga et al., 2009).

As carcaças dos animais atropelados utilizadas no estudo estavam sujeitas a diversos graus de autólise e em algumas havia o rompimento de vísceras, possivelmente devido ao impacto das colisões com os veículos. Isso pode explicar o baixo número de helmintos recuperados e a inexistência de qualquer espécie de parasita em um dos animais examinados. No entanto, Griese (2007) e Soares (2012), utilizando-se de material semelhante, descrevem uma helmintofauna diversa e com riqueza de detalhes, destacando a utilização de animais silvestres atropelados como uma excelente forma de se realizar estudos parasitológicos in situ.
Os resultados apresentados revelam três espécies de helmintos em comum entre os hospedeiros analisados, denotando possíveis relações ecológicas interespecíficas e sobreposição de distribuição na região estudada, o que contribui para a disseminação de helmintoses.

\section{CONCLUSÕES}

Caracterizou-se uma helmintofauna local e condizente com hábitos e biologia dos tamanduás examinados, recuperando-se espécies de nematódeos (Graphidiops dissimilis e Physaloptera magnipapilla), cestódeos (Mathevotaenia spp.) e acantocéfalos (Gigantorhynchus echinodiscus). A espécie Physaloptera magnipapilla foi a mais prevalente entre os helmintos recuperados, demandando mais estudos acerca do impacto de sua infecção em termos locais. Novo registro de localização foi reportado para a espécie Graphidiops dissimilis e foi detectado o compartilhamento de espécies de helmintos entre os animais analisados, sugerindo relações de distribuição e ecológicas que contribuam para a disseminação de helmintoses.

\section{REFERÊNCIAS}

AMATO, J.F.R.; CANCELLO, E.M.; ROCHA, M.M. et al. Cystacanths of Gigantorhynchus echinodiscus (Acanthocephala, Gigantorhynchidae), in Neotropical termites (Isoptera, Termitidae). Neotrop. Helminthol., v.8, p.325-328, 2014.

AMIN, O.M. Classification of acanthocephala. Folia Parasitol., v.60, p.273-305, 2013.

ANDERSON, R.C.; CHABAUD, A.G.; WILMOTT, S. Keys to the nematode parasites of vertebrates: archival volumes. Cambridge: Cabi, 2009. 463p.

BRASIL. Ministério dos Transportes. Ficha das Rodovias Brasileiras. BR-050, 2019a. Disponível em:

http://infraestrutura.gov.br/images/BIT_TESTE/

Fichas/Rodovias/Fichas_Rodovias_-_050.pdf.

Acessado em: 2 dez. 2019.

BRASIL. Ministério dos Transportes. Fichas das Rodovias Brasileiras. BR-455, 2019b. Disponível em:

$<$ http://infraestrutura.gov.br/images/BIT_TESTE

/Fichas/Rodovias/Fichas_Rodovias_-_BR-

455.pdf>. Acessado em: 2 dez. 2019. 
BUSH, A.O.; LAFFERTY, K.D.; LOTZ, J.M. et al. Parasites meets ecology on its own terms: Margolis et al. revisited. J. Parasitol., v.83, p.575583, 1997.

CAMERON, T.W.; MYERS, B.J. On a phylogenetic classification of the family trichostrongylidae leiper of marsupials, insectivores and edentates. J. Helminthol., v.35, p.25-34, 1961.

CARVALHO, C.F.; CUSTÓDIO, A.E.I; JÚNIOR, O.M. Wild vertebrates roadkill aggregations on the BR-050 highway, state of Minas Gerais, Brazil. Biosci. J., v.31, p.951-959, 2015.

CHEN, H.L.; KOPROWSKI, J.L. Barrier effects of roads on an endangered forest obligate: influences of traffic, road edges, and gaps. Biol. Conserv., v.199, p.33-40, 2016.

CLARK, J.A. Physaloptera stomach worms associated with chronic vomition in a dog in western Canada. Can. Vet. J., v.31, p.840, 1990.

DIAW, O.T. Contribution à 1'étude de nématodes Trichostrongylides parasites de Xenarthre, Marsupiaux et Rongeurs néotropicaux. Bull. Mus. Natl. Hist., v.405, p.1065-1089, 1976.

EZQUIAGA, M.C.; SUPERINA, M.; NAVONE, G.T. Parásitos intestinales de Zaedyus pichiy (Xenarthra: Dasypodidae) de Mendoza, Argentina. Mastozool. Neotrop., v.16, p.309-319, 2009.

FRANK, R.; MELAUN, C.; MARTINS, M.M.; SANTOS, A.L.Q. et al. Tunga penetrans and further parasites in the giant anteater (Myrmecophaga tridactyla) from Minas Gerais, Brazil. Parasitol. Res. v.111, p.1907-1912, 2012.

FREITAS, J.F.T.; MENDONÇA, J.M. Nota prévia sobre novo nematódeo tricostrongilídeo parasito de tamanduá-bandeira. Atas Soc. Biol. R.J., v.3, p.1-4, 1959.

GOMES, A.P.N.; CESÁRIO, C.S.; OLIFIERS, N. et al. New morphological and genetic data of Gigantorhynchus echinodiscus (Diesing, 1851) (Acanthocephala: Archiacanthocephala) in the giant anteater Myrmecophaga tridactyla Linnaeus, 1758 (Pilosa: Myrmecophagidae). Int. J. Parasitol. Parasit. Wildl., v.10, p.281-288, 2019.
GRIESE, J. Helmintofauna de vertebrados atropelados em rodovias da região de Botucatu, São Paulo. 2007. 75f. Dissertação (Mestrado em Biologia Geral e Aplicada) - Instituto de Biociências, Universidade Estadual Paulista Júlio de Mesquita Filho, Botucatu, SP.

HAMANN, O. Das system der acanthocephalen. Zool. Anz., v.15, p.195-197, 1892.

KHALIL, L.F.; JONES, A.; BRAY, R.A. Key to the cestode parasites of vertebrates. Wallingford: CAB International, 1994. 768p.

LENT, H.E.; FREITAS, J.F.T. Pesquisas helmintológicas realizadas no Estado do Pará.VI. Acantocephala. Mem. Inst. Oswaldo Cruz, v.33, p.363-380, 1938.

LÉRTORA, W.J.; MONTENEGRO, M.; MUSSART, N.B. et al. Anemia and hyperplastic gastritis in a giant anteater (Myrmecophaga tridactyla) due to Physaloptera magnipapilla Parasitism. Braz. J. Vet. Pathol., v.9, p.20-26, 2016.

LUNASCHI, L.I.; LAMAS, M.F.; DRAGO, F.B. A new species of Mathevotaenia (Cestoda, Anoplocephalidae) parasitizing Tropidurus spinulosus (Reptilia, Squamata) from northeastern Argentina. Rev. Mex. Biodivers., v.83, p.583-590, 2012.

LYMBERY, A.J. Parasites and ecosystem health. Int. J. Parasitol., v.35, p.703, 2005.

MACHADO FILHO, D.A. Sobre alguns Acantocéfalos do Estado do Pará. Rev. Bras. Biol., v.1, p.223-226, 1941.

MEDRI, I.M.; MOURÃO, G.M.; RODRIGUES, F.H.G. Ordem pilosa. In: REIS, N.R.; PERACCHI, A.L.; PEDRO, W.A.; LIMA, I.P. (Eds.). 2.ed. Mamíferos do Brasil. Londrina: Technical Books, 2011. p.91-101.

MIRANDA, F. Cingulata (tatus) e Pilosa (Preguiças e tamanduás). In: CUBAS, Z. S.; SILVA, J.C.R.; CATÃO-DIAS, J.L. (Eds.). 2.ed. Tratado de animais selvagens. São Paulo: Roca, 2014. p.707-722.

MIRANDA, F.; CHIARELLO, A.G.; ROHE, F. et al. Avaliação do risco de extinção de Xenartros brasileiros. Brasília, DF: ICMBio, 2015. 250p.

ORTLEPP, M.A.The Nematode genus Physaloptera rudolphi, 1819. Proc. Zool. Soc. Lond., v.2, p.999-1107, 1922. 
PINTO, F.A.S.; BAGER, A.; CLEVENGER, A.P. et al. Giant anteater (Myrmecophaga tridactyla) conservation in Brazil: Analysing the relative effects of fragmentation and mortality due to roads. Biol. Conserv., v.228, p.148-157, 2018.

RAMOS, D.G.S.; SANTOS, A.R.G.L.O.; FREITAS, L.C. et al. Endoparasites of wild animals from three biomes in the State of Mato Grosso, Brazil. Arq. Bras. Med. Vet. Zootec., v.68, p.571-578, 2016.

ROJANO, C.; MIRANDA, C.L.; RENZO, A.A. Endoparasitos de Myrmecophaga tridactyla y Tamandua tetradactyla (pilosa: vermilingua) silvestres en Casanare, Colombia. Rev. Colomb. Cienc. Anim., v.7, p.154-159, 2015.

ROSA, R.; LIMA, S.C.; ASSUNÇÃO, W.L. Abordagem preliminar das condições climáticas de Uberlândia (MG). Soc. Nat., v.3, p.91-108, 1991.

SCHMIDT, G.D.; MARTIN, R.L. Tapeworms of the Chaco Boreal, Paraguay, with two new species. J. Helminthol., v.52, p.205-209, 1978.

SCHMIDT, G.D. Handbook of tapeworm identification. Florida: CRC Press, 1986. 675p.

SOARES, R. Helmintofauna de Procyon cancrivorus (G. [Baron] Cuvier, 1798), Nasua nasua (Linnaeus 1766) e Galictis cuja (Molina, 1782), atropelados na BR-116 - Rio de Janeiro Teresópolis - Além Paraíba, RJ, Brasil. 2012. 136f. Dissertação (Mestrado em Microbiologia e Parasitologia Aplicadas) - Departamento de Microbiologia e Parasitologia, Universidade Federal Fluminense, Rio de Janeiro, RJ.
SPASSKII, A.A. Anoplocephalate tapeworms of domestic and wild animals. In: SKRJABIN, K.I. (Ed.). Essentials of cestodology. Moscow: Academy of Sciences of the USSR, 1951. p. 15634.

SUPERINA, M.; MIRANDA, F.; ABBA, A.M. The anteater red list assessment. Edentata, v.11, p.96-114, 2010.

TRAVASSOS, L. Contribuição ao conhecimento dos Trichostrongylidae de Tamandua tetradactyla (L.) (Nematoda). An. Inst. Biol., v.20, p.251-269, 1949.

VALADÃO, M.; BASTOS, L.F.; CASTRO, C.P. Atropelamento de vertebrados silvestres em quatro rodovias no Cerrado, Mato Grosso, Brasil. Multi Sci. J., v.1, p.62-74, 2018.

VICENTE, J.J.; RODRIGUES, H.O.; GOMES, D.C. et al. Nematoides do Brasil. Parte V: Nematoides de mamíferos. Rev. Bras. Zool., v.14, p.1-452, 1997.

YAMAGUTI, S. Systema helminthum. London: Interscience Publishers, 1963. 423p. (v.5, Acanthocephala). 